$\xi=2$ 줄

\title{
A comparative study to diagnose the accuracy of E-speed film, complimentary metal oxide semiconductor and storage phosphor systems in the detection of proximal caries: An in vitro study
}

\author{
Tatapudi Ramesh ${ }^{1}$, Gurugubelli Upendra ${ }^{2} *$, Bandaru Sravani Krishna ${ }^{2}$, Sahithi Dathar ${ }^{2}$, \\ Priyankesh $^{2}$, Raghavendra $\mathrm{MN}^{3}$, Swathi Myla ${ }^{2}$, Roja. K ${ }^{2}$ \\ ${ }^{1}$ Professor, Department of Oral Medicine and Radiology \\ ${ }^{2}$ Post Graduate student, Department of Oral Medicine and Radiology \\ ${ }^{3}$ Reader, Department of Oral Medicine and Radiology \\ *Corresponding author E-mail: upendra.regals@gmail.com
}

\begin{abstract}
Background: Dental caries is one of the most commonly encountered conditions in clinical dentistry and these lesions remain undetected when confined to the vicinity of inter-proximal surfaces. Radiography plays a key role in the detection of inter-proximal caries especially in tight contacts.

Objectives: The purpose of this study was to compare the diagnostic accuracy of E-speed film, complementary metal oxide semiconductors (CMOS) and storage phosphor systems (PSP) in the detection of proximal caries of the posterior teeth.

Methods: Conventional films, CMOS and PSP images were used in detecting proximal caries on mesial and distal surfaces of 63 teeth (126 surfaces). Interpretation of all digital and conventional radiographs were performed and reanalyzed by four observers. The collected data was subjected to statistical analysis using chi square test, weighed kappa statistics and spearman rank correlation coefficient.

Results: The PSP images showed more accurate results in identifying normal tooth, enamel caries, dentinal caries and deep dental caries and kappa statistics had represented almost perfect reading of $0.8-0.9$ for PSP images whereas CMOS images showed substantial reading of $0.6-0.7$, and for IOPA images it showed moderate reading of $0.5-0.6$, which stated that the higher inter-observer agreement was obtained for PSP images when compared with images taken by IOPA and CMOS. The intra-observer reliability by kappa statistics had shown highly significant value $(0.82)$ in the present study.

Conclusion: Conventional films, CMOS and PSP images had shown almost appropriate results in the detection of proximal caries but PSP receptors were better in disclosing the details more accurately in terms of delineating the actual extent of the lesion pertaining to their high resolution capacity and further their flexibility made them easier during handling the radiograph, when compared with that of rigid CMOS receptors.
\end{abstract}

Keywords: Proximal caries; Film; CMOS; PSP

\section{Introduction}

Dental caries is one of the most regularly encountered conditions in clinical dentistry. Among them Inter-proximal carious lesions, which develop between the contacting surfaces of two adjacent teeth were often missed during routine clinical examination. They appear as opaque regions clinically which are caused by loss of enamel translucency at the outermost portion of enamel, between the contact points and just coronal of the free gingival margins. Currently, clinical examination of these lesions, even under ideal state of affairs results in an unacceptable proportion of false negative results, especially in the areas of tight contact points that hamper visual inspection.

The greatest difficulty in detecting the carious lesions is experienced in their initial stages, when confined to the enamel layer. With the intention to increase the frequency of detection of these proximal caries, several diagnostic methods are being explored till date, they include trans-illumination, laser fluorescence, electrical impendence measurement, digital imaging and intraoral cameras (Ludlow et al.2004, Erten et al.2006).

Developments have made it viable to use latest computer technologies to acquire and display digital radiographic images. With the advent of digital radiography in current dental practice several novel digital radiographic systems had substituted the conventional film based radiography. Many studies shown that digital systems have few advantages when compared with conventional film such as reduced exposure dose, reduced processing artifacts, reduced working time from image exposure to image display, image communication is easier and increased diagnostic accuracy and no contamination with processing solutions and less processing errors(Kamburoglu et al 2010).

In direct digital image acquisition two different modalities are used which include Solid state detector system like Charge coupled devices (CCD) that uses a thin wafer of silicon for image production and Complementary metal oxide semiconductor (CMOS) which has a light sensitive chip with a scintillation detec- 
tor. In indirect digital image acquisition storage phosphor system (PSP plate) were used which consists of polyester base coated with crystalline halide composed of europium-activated barium fluorohalide compounds. By using these systems, captured information is converted to electrical signals, which were subsequently digitalized and final image will be displayed (Kamburoglu et al 2010, Wenzel 1998).

In view of the importance of radiological diagnosis of proximal caries and the potential difference in diagnostic performance of different caries detection methods, the aim of present study was to assess diagnostic accuracy and user friendly method among conventional and digital radiographs in evaluation of proximal caries.

\section{Materials and methods}

A cross sectional in vitro study was carried out on 63 human premolar and molar teeth with proximal caries, which have been extracted for various periodontal and orthodontic reasons. Carious surfaces with varying degree of demineralization which appear as chalky white or brownish discoloration on proximal surfaces were included in the present study. Those teeth with restorations on proximal surfaces, extensive buccal or lingual caries, dental wear, presence of fractures or anomalies, grossly decayed or with deep dental caries in proximal surfaces were excluded from the study and the study protocol was approved by institutional ethical committee.

Teeth were cleaned to remove calculus and debris and were disinfected in $2 \%$ sodium hypochlorite solution for about 20 minutes and stored in distilled water. Then these teeth were embedded in blocks of dental stone with three teeth in each block ensuring to maintain the contact between the proximal surfaces and each block was assigned a number. The mesial and distal surfaces of the teeth in contact were assessed for presence of proximal caries Detection of proximal caries was performed using visual examination, conventional E- speed film, CMOS and PSP images.

All blocks were imaged using three intraoral periapical receptors Under standardized conditions at $60 \mathrm{kvp}, 8 \mathrm{~mA}$ and $0.2 \mathrm{sec}$ for Espeed film with $10 \mathrm{~mm}$ tooth receptor distance, $30 \mathrm{~cm}$ target-toreceptor distance using rectangular collimation and paralleling technique were used. A $10 \mathrm{~mm}$ thick acrylic block was placed behind the teeth in each block in order to stimulate the soft tissue. After exposure all the films were simultaneously developed by using developer and fixer solution, according to the instructions recommended by the manufacturer. For digital radiographic exposure, all adjustments for teeth and radiography apparatus were done similar to that of E-speed film, but the only difference was the exposure time which was reduced to approximately $0.08 \mathrm{sec}-$ ond.

All images were evaluated separately by four oral and maxillofacial radiologists who were experienced in interpretation of radiograph atleast for a period of 5 years mainly to reduce the bias and there was no restriction of time for the observers.

The presence or absence of the proximal caries was assessed according to radiographic criteria using following scale: 0 :- no caries detected in the proximal surface; 1:- surface with caries involving only enamel; 2:- surface with caries involving up to the dentinoenamel junction; 3:- surface with caries extending up to dentin. The obtained data was subjected to statistical analysis. Spearman rank correlation coefficient was designed for inter group comparison and kappa statistics was considered to assess Inter and Intraobserver reliability by observing all images and reanalyzing with a 2 week interval between observations so as to eliminate memory bias and was weighed up according to the following criteria of kappa statistics: $0.4-0.6$, moderate reading; $0.6-0.8$, substantial reading; $0.8-1.0$, almost perfect reading.

\section{Results}

A total of 63 teeth with three teeth on each radiograph were evaluated. Table 1 shows actual extent of the lesion involving enamel, upto the dentino-enamel junction and into the dentine using chi square test. The presence of the lesion is equally distributed on both PSP (66) and CMOS (66) images which means that the lesion is more accurately visible in digital receptor and presence of initial caries is more appreciated in PSP (25) than in CMOS (23) and IOPA (21) which shows that PSP is more accurate in detecting early lesions and the deeper lesions were more visible in IOPA images (11) in comparison with CMOS (7) and PSP (6) which means IOPA's have a slight less ability to detect the presence of initial forms of caries and also in delineating the actual depth of the caries.

Table 1: Exact Presence of the Lesion

\begin{tabular}{llll}
\hline LESION PRESENCE & IOPA $(\mathrm{n})$ & CMOS(n) & PSP(n) \\
\hline SOUND TOOTH & 68 & 66 & 66 \\
ENAMEL CARIES & 21 & 23 & 25 \\
DEJ & 26 & 28 & 32 \\
DENTINAL CARIES & 11 & 7 & 6 \\
\hline
\end{tabular}

Table 2 Shows Inter-group comparison in detecting the presence of carious lesion using spearman rank correlation coefficient which shows positive correlation exists between all the groups and was statistically significant $(p \leq 0.05)$. Subsequently, the presence of caries was almost equal in each group.

Table 2: Inter Group Comparison

\begin{tabular}{llll} 
RADIOGRAPH & $\mathrm{N}$ & CORRELATION COEFFICIENT & p-value \\
\hline IOPA & 126 & 1.000 & 0.000 \\
CMOS & 126 & 0.958 & \\
IOPA & 126 & 1.000 & 0.000 \\
PSP & 126 & 0.951 & \\
CMOS & 126 & 0.958 & 0.000 \\
PSP & 126 & 0.951 & \\
\hline
\end{tabular}

Table 3 Showed interobserver comparison using kappa coefficients which were calculated for each observer. Higher interobserver agreement was obtained from the PSP images when compared with CMOS and IOPA images. Inter-observer kappa coefficients ranged from 0.5 - 0.6 for the IOPA images, from 0.6 0.7 for the CMOS images, and from $0.8-0.9$ for the PSP images, suggesting strong and excellent inter observer agreement in general and PSP images represented almost perfect (AP) reading, CMOS images showed substantial (S) reading, and IOPA images showed moderate reading.

Table 3: Inter Observer Comparison

\begin{tabular}{lc}
\hline IOPA & 0.59 \\
\hline CMOS & 0.67 \\
PSP & 0.81 \\
\hline
\end{tabular}

Table 4 Shows intraobserver kappa coefficients and was found to be highly significant $(0.82)$.

Table 4: Intra Observer Comparison

\begin{tabular}{ll} 
IOPA & \\
CMOS & 0.82 \\
PSP & \\
\hline
\end{tabular}

\section{Discussion}

Dental caries is most frequently observed condition in clinical dentistry and different methods have been proposed till date to detect the presence of caries and also to delineate actual extent of the caries. One such method was by obtaining a radiographic image of the carious lesion. Several studies were performed and their results revealed that about 25 to $42 \%$ of these lesions remain undetected by clinical examination alone without radiographic inter- 
vention (Senel et al.2010). Dental caries confined to the interproximal areas were failed to spot out during routine clinical examination because of tight contacting surfaces.

The accurate diagnosis of the presence of disease is of paramount significance to provide appropriate care. Radiographic methods have been a potential investigatory method since decades but do have a potential risk of ionizing radiation, so if exposure level used to obtain a radiographic image is minimized, it may be advantageous for the patient (Hintze et al.2002). So intra oral periapical radiographs were most commonly prescribed method for evaluating dental caries. In this modality conventional radiographs were widely used for a number of diagnostic tasks to provide accurate information. In recent times as a part of improvising the existing image obtaining methods these conventional forms were digitized with receptors like CCD, CMOS, storage phosphor system and one should expect diagnostic accuracy of them which is supposed to be more or at least to that of a film.

In the present study there is no statistically significant difference observed between the digital systems and conventional films while diagnosing the presence of inter-proximal caries. Several studies have measured that diagnostic ability of intraoral digital sensors and the conventional film in the detection of carious lesions. Senel B et al, Pontual AA et al, White SC and Yoon DC conducted studies and compared conventional films and digital sensors in detecting the proximal caries and revealed that no statistically significant difference exists, which are in accordance with present study (Senel B et al.2010, White SC and Yoon DC.1997, Peker et al.2009, Pontual et al.2010). Abesi F et al, conducted a study and results obtained showed that conventional film is more accurate in detecting the proximal caries, which is in contradictory to present study (Abesi et al.2012).

In present study, teeth with more superficial dental caries were preferred, after clinical examination. If diagnostic discrepancies between radiographic systems were to be found, their accuracy in detecting subtle pathological changes must be examined. The present study observed that the accuracy in detecting superficial lesions on proximal surfaces was found to be highest in storage phosphor images (25), followed by CMOS (23) and IOPA films (21) but it is statistically insignificant. Similar result was obtained with the work done by $\mathrm{Li}$ et al, even if the initial caries present on the proximal surfaces they are not radiographically detected and deeper lesions were more easily detected than the superficial ones (Wenzel et al.1998).

According to Pereira AC et al, a radiograph was unable to detect initial occlusal enamel and dentin lesions, resulting in low sensitivity which is analogous to results of present study (Pereira et al.2009). In accordance with present study, studies conducted by Syrioupoulos et al and White and Yoon revealed deeper caries lesions were easier to detect using radiographic systems than relatively superficial ones (White SC and Yoon DC.1997, Syriopoulos et al.2000). As the lesions penetrate dentine, observers were able to detect their presence more consistently. Clinical diagnosis of enamel caries was even harder to perform than radiographic diagnosis.

The number of examiners was another fruitful factor. Bader et al believed that in some studies which evaluate the methods for caries detection with small number of observers is a limiting factor (Bader et al.2001). So the present study included 4 observers, who were experienced in interpretation of radiograph for atleast a period of 5 years. This was mainly done so as to reduce the observer bias.

In present study, both digital and conventional radiographic modalities were used to detect proximal caries showed that there is no statistically significant difference between these methods. In correspondence with the study conducted by Aberu et al on diagnostic accuracy of CCD (RVG UI) digital radiography and Ekta Speed plus film radiographs to determine interdental caries, it revealed that the ability of two imaging systems were not statistically significant (Abreu et al. 2001).

Among various researches performed on carious lesions, the studies conducted by Pontual et al, Haiter-Neto et al, on PSP plates showed similar results to that of the film for detection of proximal enamel caries which is similar to the present study (Pontual et al, 2010, Haiter-Neto et al. 2008).

In present study the intergroup comparison shows the positive correlation exists between all the groups that is when comparing IOPA with CMOS, CMOS with PSP and PSP with IOPA groups where $p$-value is $\leq 0.05$ and the presence of caries is almost equal in each group which is similar to study conducted by Pontual AA et al, wherein no significant difference was observed in diagnostic accuracy among the insight film and Digora and Denoptix digital systems for proximal enamel caries (Pontual et al. 2010). Study conduted by Abesi et al, also demonstrated that no significant difference between digital and conventional radiographic modalities in detection of interproximal caries (Abesi et al.2012).

A very high Interobserver kappa coefficients found in the present study suggested excellent interobserver agreement and strong intraobserver agreement among PSP images with intraobserver kappa coefficient range at 0.82 . Interobserver kappa coefficient ranges from $0.5-0.6$ for the IOPA images, from $0.6-0.7$ for the CMOS images, and from $0.8-0.9$ for the PSP images.

Research done by Senel B et al showed excellent interobserver agreement (kappa coefficient 50.89) with a kappa coefficient of 0.79 and reported that intraobserver agreement using bitewing film in an in vitro detection of proximal caries (Senel et al.2010).

The differences in intra and interobserver agreement kappa values among the different studies may be related to observer experience, radiographic quality, viewing conditions, study design and study material, all of which are important factors in determining observer agreement (Senel et al.2010, Rocha et al.2005, Tantanapornkul et al.2012).

The manipulation of digital images is another variable that differs in works that compare digital systems to conventional film radiographs. This study was designed to simulate clinical conditions as much as possible, where the observer was allowed to alter the brightness, contrast and size, as well as reverse the image. Another reason was the possibility of enhancing the image and compensating the lower resolution in the digital systems, thereby obtaining an image of good diagnostic quality (Rockenbach et al.2008).

According to the guidelines of the American Dental Association (2006), radiographic diagnosis should only be used after clinical examination, considering the dental and general health needs of the patient. Since situations for each patient differs, radiographic examination should be individualized and should consider the initial routine dental examination because the hidden caries can be detectable only using radiographically in some patients. So radiography plays crucial role in diagnosis of initial and occult dental caries (Torres et al.2011).

Storage phosphor technology provides film-like sensors which are flexible and easier to place for all intraoral imaging sites. It also adds up some of the advantages over conventional films such as: No-processing problems like darkroom, processor and processing chemistry not required (environmentally friendly), less exposure errors like density and contrast, enhancement, it also allows for quantitative evaluation and lower absorbed doses. Teleradiography also helps in transmission of digital images to remote sites which have been a major driving force in the evolution of digital radiograph as well.

In present study when E-speed films, CMOS and PSP receptors were compared, their ability to detect caries was almost similar, but actual extent of caries extent is better appreciated in digital receptors and when CMOS and PSP receptors are compared, PSP receptors are better in disclosing the presence of proximal caries.

\section{Conclusion}

In the present study, the diagnostic accuracy of all the three intraoral modalities was observed to be almost similar in detection of proximal caries. But, extent of involvement is better appreciated in digital radiographs. On comparison of CMOS and PSP digital receptors, performance of PSP receptors showed the actual 
extent of the lesion and was better in disclosing the details more accurately owing to their high spatial resolution capacity. Thus, the present study had tried to uncover the hidden aspects for the detection of occult proximal dental caries by means of three different intraoral radiographic modalities and further ease in application due to the flexibility of PSP receptors when compared to rigid CMOS receptors was also observed in the current study.

\section{References}

[1] Abesi F, Mirshekar A, Moudi E, Seyedmajidi M, Haghanifar S, Haghighat N, Bijani A.(2012) Diagnostic Accuracy of Digital and Conventional Radiography in the Detection of NonCavitatedApproximal Dental Caries. Iran J Radiol. 9:17-21. http://dx.doi.org/10.5812/iranjradiol.6747.

[2] Abreu M, Mol A, Ludlow JB. (2001) Performance of RVGui sensor and Kodak Ektaspeed Plus film for proximal caries detection. Oral Surg Oral Med Oral Pathol Oral RadiolEndod. 91:381-5 http://dx.doi.org/10.1067/moe.2001.112393.

[3] Bader JD, Shugars DA, Bonito AJ. (2001) Systematic Reviews of Selected Dental Caries Diagnostic and Management Methods Journal of Dental Education.65:960-8

[4] Erten H, Uctasli MB, Akarslan ZZ, Uzun O, Semiz M.(2006) Restorative Treatment Decision Making with Unaided Visual Examination, Intraoral Camera and Operating Microscope. Operative Dentistry, 3:55-59 http://dx.doi.org/10.2341/04-173.

[5] Haiter-Neto F, Wenzel A and Gotfredsen E. (2008) Diagnostic accuracy of cone beam computed tomography scans compared with intraoral image modalities for detection of caries lesions. Dentomaxillofacial Radiology.37:18-22 http://dx.doi.org/10.1259/dmfr/87103878.

[6] Hintze H, Wenzel A and Frydenberg M. (2002) Accuracy of caries detection with four storage phosphor systems and E-speed radiographs. Dentomaxillofacial Radiology.31:170-175 http://dx.doi.org/10.1038/sj.dmfr.4600686.

[7] Kamburoglu K, Senel B, Yuksel SP and Ozen T. (2010) A comparison of the diagnostic accuracy of in vivo and in vitro photostimulable phosphor digital images in the detection of occlusal caries lesions. Dentomaxillofacial Radiology. 39:17-22. http://dx.doi.org/10.1259/dmfr/91657756.

[8] Ludlow JB, Mol A. Digital imaging. In: White SC, Pharoah M (eds). (2004) Oral radiology: Principles and interpretation (5th ed). St.Louis: Mosby. 225-244.

[9] Pereira AC, Eggertsson H, Moustafa A, Zero DT, Eckert GJ, Mialhe FL.(2009) Evaluation of three radiographic methods for detecting occlusal caries lesions.Braz J Oral Sci.8(2):67-70

[10] Peker I, ToramanAlkurt M, Usalan G, Altunkaynak B. (2009) the comparison of subjective image quality in conventional and digital panoramic radiography. Indian J Dent Res 20:21-5 http://dx.doi.org/10.4103/0970-9290.49052.

[11] Pontual AA, de Melo DP, de Almeida SM, Boscolo FN and HaiterNeto F. (2010) Comparison of digital systems and conventional dental film for the detection of approximal enamel caries. Dentomaxillofacial Radiology. 39:431-436 http://dx.doi.org/10.1259/dmfr/94985823.

[12] Rocha A S P S da, Almeida S M de, Boscolo F N, HaiterNeto F.(2005)Interexaminer agreement in caries radiographic diagnosis by conventional and digital radiographs. J Appl Oral Sci.13:329-33 http://dx.doi.org/10.1590/S1678-77572005000400003.

[13] Rockenbach M.I, Veeck EB, Pereira da Costa N.(2008) Detection of proximal caries in conventional and digital radiographs: an in vitro study. Stomatologija, Baltic Dental and Maxillofacial Journal. 10:115-120

[14] Senel B, Kamburoglu K, Ucok O, Yuksel SP, Ozen T and Avsever H. (2010) Diagnostic accuracy of different imaging modalities in detection of proximal caries. Dentomaxillofacial Radiology.39:501-511 http://dx.doi.org/10.1259/dmfr/28628723.

[15] Syriopoulos K, Sanderink GCH, Velders XL and van der Stelt PF.(2000) Radiographic detection of approximal caries: a comparison of dental films and digital imaging systems. Dentomaxillofacial Radiology.29:312-318 http://dx.doi.org/10.1038/si.dmfr.4600553.

[16] Tantanapornkul W, Mongkolrop P, Manoping P, Hannanta-anan A, Prompruk E.(2012) Efficacy of Different Image Processing Programs in Detecting Small Artificial Caries Defects on Approximal Surfaces. Dentistry. 2:1-5. http://dx.doi.org/10.4172/21611122.1000129

[17] Torres MGG, Santos AS, Neves FS, Arriaga ML, Campos PSF Crusoe-rebello I.(2011) Assessment of enamel-dentin caries lesions detection using bitewing PSP digital images. J Appl Oral Sci. 19:462-8

[18] Wenzel, A. (1998) Digital radiography and caries diagnosis: a review. Dentomaxillofacial Radiology. 27:3-11. http://dx.doi.org/10.1038/sj.dmfr.4600321.

[19] White SC and Yoon DC. (1997) Comparative performance of digital and conventional images for detecting proximal surface caries. Dentomaxillofacial http://dx.doi.org/10.1038/sj.dmfr.4600208.
Radiology.26:32-31. 